\title{
Angle-range-polarization-dependent beamforming for polarization sensitive frequency diverse array
}

\author{
Hui Chen ${ }^{1^{*}}$ (D), HuaiZong Shao ${ }^{1}$ and HongYang Chen ${ }^{2}$
}

\begin{abstract}
In this paper, we present a polarization sensitive frequency diverse array (PSFDA), which benefits both from frequency diverse array (FDA) and polarization sensitive array (PSA), producing angle-range-polarization-dependent beamforming capability. To simultaneously improve the angle, range and polarization resolution, the $\ell_{1}$-norm minimization-based sparse constraint and $\ell_{\infty}$-norm constraint for sidelobe region are further applied. Besides, the performance of the optimal output signal-to-interference-plus-noise ratio (SINR) is analyzed for such a high-dimensional arrays. Numerical results demonstrate that the proposed PSFDA provides both range and polarization resolution as well as target angle estimation. Moreover, the PSFDA virtually increases the array size by exploiting multiple electromagnetic fields at each physical point. In addition, the proposed beamforming method can effectively null interferences, without degrading the array gain for the signals of interest.
\end{abstract}

Keywords: Array signal processing, FDA, PSA, Beamforming, Sparse constraint, Convex optimization

\section{Introduction}

Multi-domain signal processing can improve the array performance by exploiting space, time, polarization, frequency resources [1-3]. Nevertheless, the range domain information has not been sufficiently explored in literature. Although existing interference suppression approaches can be employed to exploit the angle as well as polarization domain information for polarization sensitive array (PSA), the joint information between range domain and other domains are rarely explored [4].

Recently, a new kind of array named frequency diverse array (FDA), which applies a small frequency increment between contiguous two elements, has gained substantial interest [5-8]. This small frequency increment, as compared to the carrier frequency, results in a anglerange-polarization-dependent beampattern [9-12] that is different from conventional phased-array providing only angle-dependent beampattern and frequency scanning array whose beam pointing angle changes with working

*Correspondence: huichen0929@uestc.edu.cn

'School of Information and Communication Engineering, University of Electronic Science and Technology of China, Xiyuan Road, Chengdu, 611731 , People's Republic of China

Full list of author information is available at the end of the article frequency. These characteristics make the FDA attractive for a wide range of applications in target imaging [13], localization [14], and bistatic radar [15-18]. Instead of using a fixed frequency offset, W. Khan et al. presented a variational frequency offset scheme to achieve time-independent but angle-range-dependent beampattern $[19,20]$. In [21], the authors derived the FDA CramérRao lower bounds for estimating target direction, range and velocity. The work about the joint applications of FDA and multi-input and multi-output (MIMO) radar can be found in [16], where the FDA elements radiate coherent waveforms, rather than orthogonal waveforms like an MIMO radar. Additionally, an analytical investigation of FDA multipath characteristics was carried out in [22]. The authors of [23] extended the linear FDA to planar array.

In this paper, to further enhance the interference mitigation capability, we propose a PSFDA by combining the PSA and the FDA. A angle-range and polarizationdependent beamforming algorithm is also proposed by jointly using the norm-function constraint theory [24] and convex optimization [25]. The proposed method can effectively suppress both clutter and interference that is not accessible for the conventional phased-array systems. The new contributions of this work is different from our 


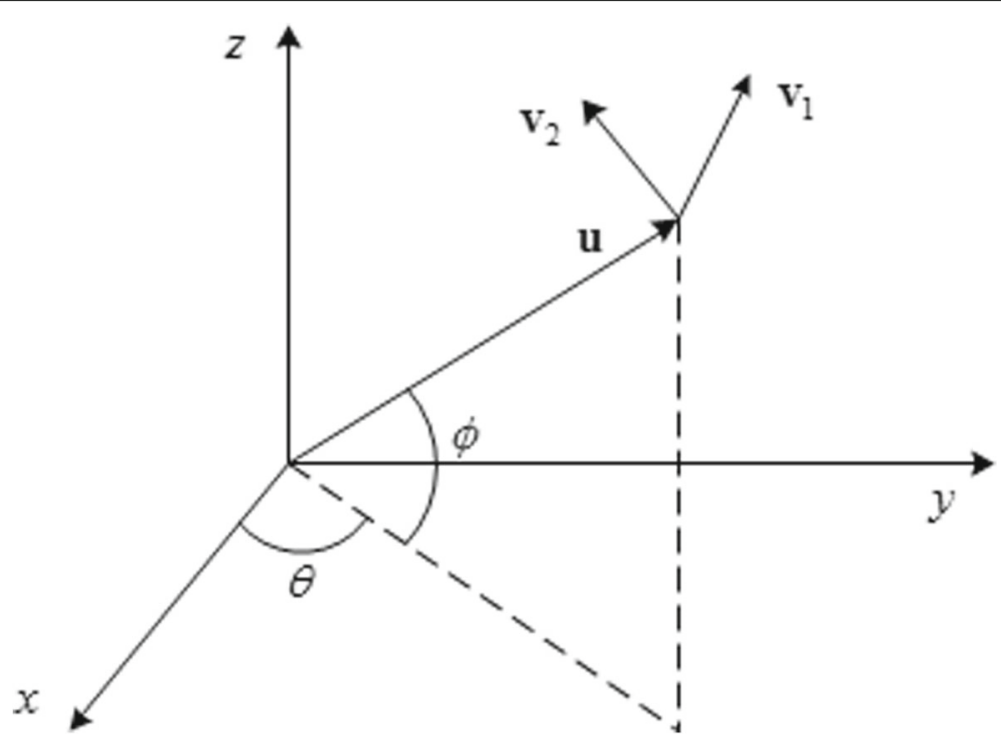

Fig. 1 Orthogonal vector triad $\left(\mathbf{u}, \mathbf{v}_{1}, \mathbf{v}_{2}\right)$

previous work [26] in at least two aspects: One is that, a more general signal model is presented for the PSFDA: the PSFDA considered in [26] is just arranged with uniformly spaced orthogonal dipole, while the PSFDA considered in this work is constituted by distributed collocated electromagnetic vector sensor (EMVS), and the antenna elements in each EMVS with $2 \leq P \leq 6$ dipole elements, which contains the special case in [26]. Therefore, the signal model of the PSFDA in this work is more general when compared to the one in [26]; The second is that the output
SINR performance of the high-dimensional PSFDAs are analyzed and simulated in a much more extensive way.

Note that, $[\cdot]^{T}$ and $[\cdot]^{H}$ denote the transpose and conjugate transpose operators, respectively. $\|\cdot\|_{1},\|\cdot\|_{2}$, and $\|\cdot\|_{\infty}$ indicate the $\ell_{1}$-norm, $\ell_{2}$-norm, and $\ell_{\infty}$-norm of a vector or matrix.

The remainder of this paper is organized as follows. Section 2 introduces the PSFDA, along with its measurement model. Section 3 proposes the angle-range and polarization dependent beamforming algorithm. Next,

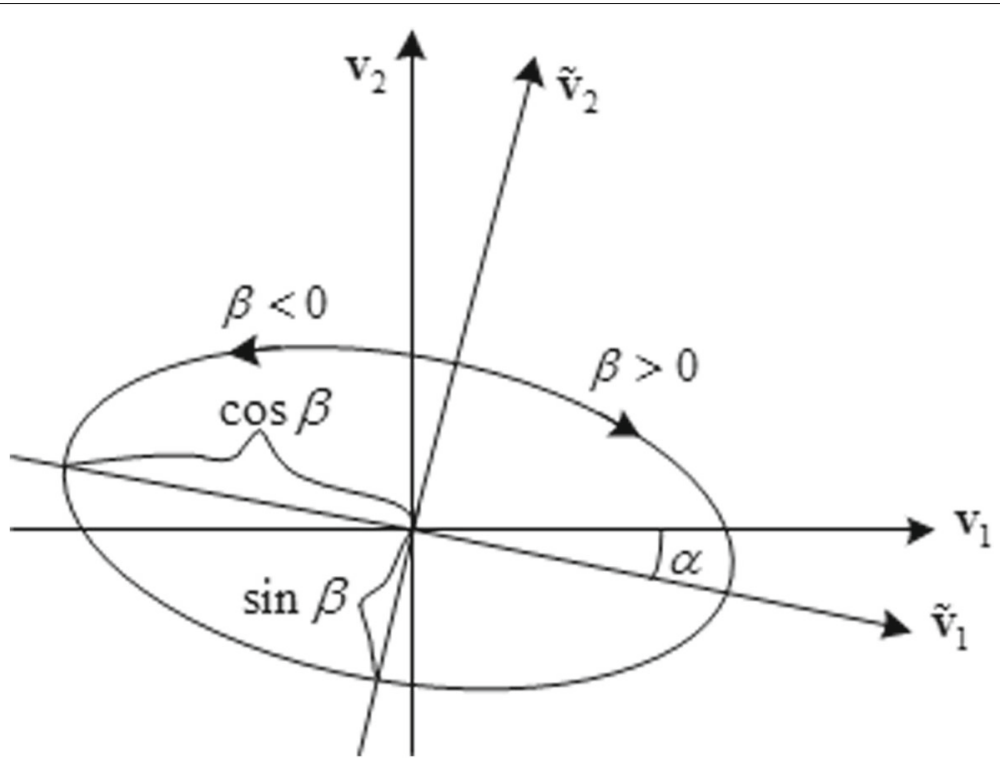

Fig. 2 Electric polarization ellipse 


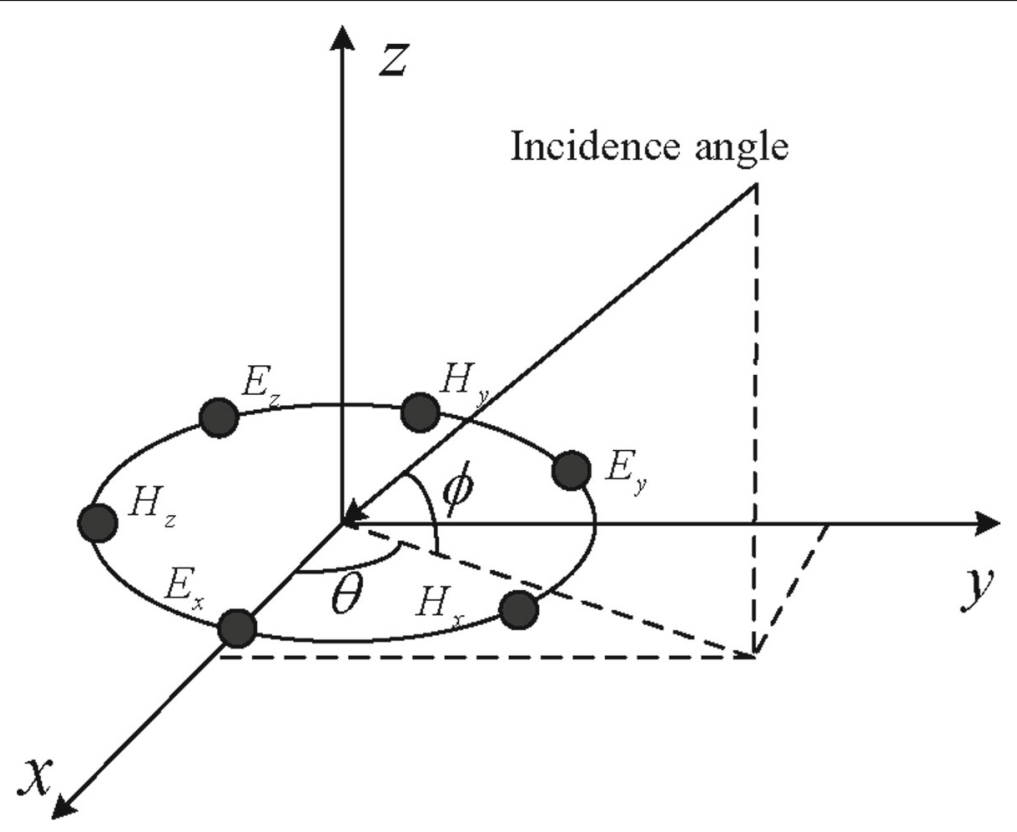

Fig. 3 Distributed electromagnetic vector sensor, where $E_{x}\left(H_{x}\right), E_{y}\left(H_{y}\right)$, and $E_{z}\left(H_{z}\right)$ are the electric (magnetic) component sensors, respectively

numerical simulation results and discussions are given in Section 4. Finally, conclusions are drawn in Section 5.

\section{PSFDA and its signal model}

\subsection{Frequency diverse array}

Consider a uniform $M$-element linear FDA with the element spacing $d$. The radiated frequency of the $m$-th element is given by [5]

$$
f_{m}=f_{0}+\Delta f_{m}, m=1,2, \ldots, M,
$$

where the frequency offset between the $m$-th element and the reference element is $\Delta f_{m}=(m-1) \cdot \Delta f, f_{0}$, and $\Delta f$ represent the carrier frequency and the frequency increment, respectively. Taking the first element as the reference for the array, then the phase difference between the $m$-th element and the reference element can be expressed as

$$
\begin{aligned}
\Delta \psi_{m-1}= & \psi_{m}-\psi_{1} \\
\approx & -\frac{2 \pi f_{0}(m-1) d \sin \theta}{c}+\frac{2 \pi r(m-1) \Delta f}{c} \\
& -\frac{2 \pi(m-1)^{2} \Delta f d \sin \theta}{c}
\end{aligned}
$$

where the approximation $r_{m} \approx r-(m-1) d \sin \theta$, where $\theta, r$, and $c$ are the direction and range of the given far-field

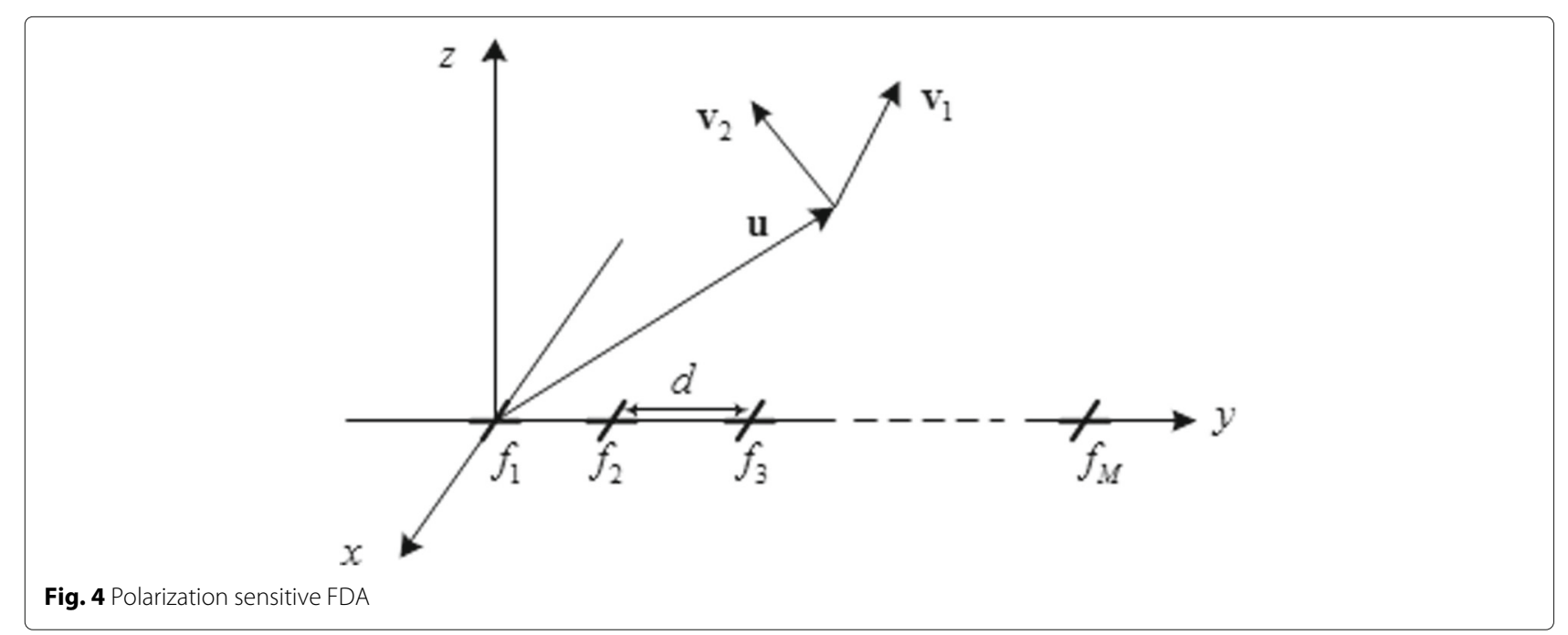



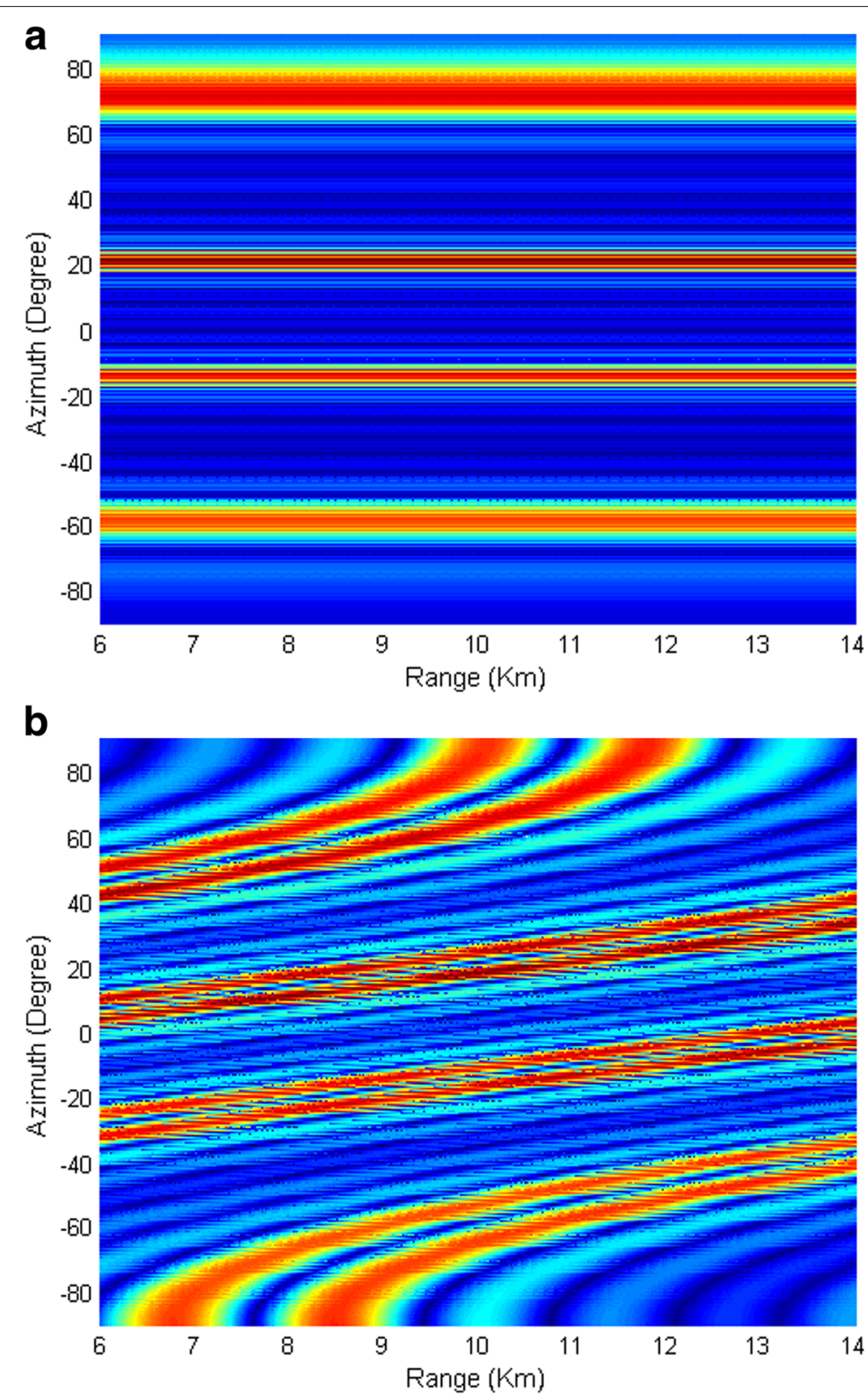

Fig. 5 Comparative beampattern between PSA and PSFDA, where $\Delta f=30 \mathrm{kHz}, M=8$, and $f_{0}=2 \mathrm{GHz}$. a PSA beampattern. b PSFDA beampattern

point and the speed of light, respectively. The steering vector of a standard FDA can be expressed as (3), if the third term of (2) is ignored due to the fact that $f_{0} \gg \Delta f$ and $r \gg d \sin \theta$, we can obtain the steering vector of FDA as

$$
\begin{aligned}
& \mathbf{a}_{f d a}(\theta, r)= \\
& \quad\left[1 e^{-j\left(\frac{2 \pi f_{0} d \sin \theta}{c}-\frac{2 \pi r \Delta f}{c}\right)} \ldots e^{-j\left(\frac{2 \pi f_{0}(M-1) d \sin \theta}{c}-\frac{2 \pi r(M-1) \Delta f}{c}\right)}\right]^{T} .
\end{aligned}
$$

It is seen that the FDA generates a range-dependent beampattern that is different from a phased-array. Therefore, FDA provides a more flexible beam scan option in either transmission or receive mode. The FDA beam direction will vary as a function of range $r$ and angle $\theta$, even for a fixed $\Delta f$. Specifically, the FDA is just a conventional phased-array when $\Delta f=$ $0 \mathrm{~Hz}$. If the range $r$ is fixed, the beam direction will vary as a function of $\Delta f$. Therefore, the angle-rangedependent beampattern of FDA provides a poten- 

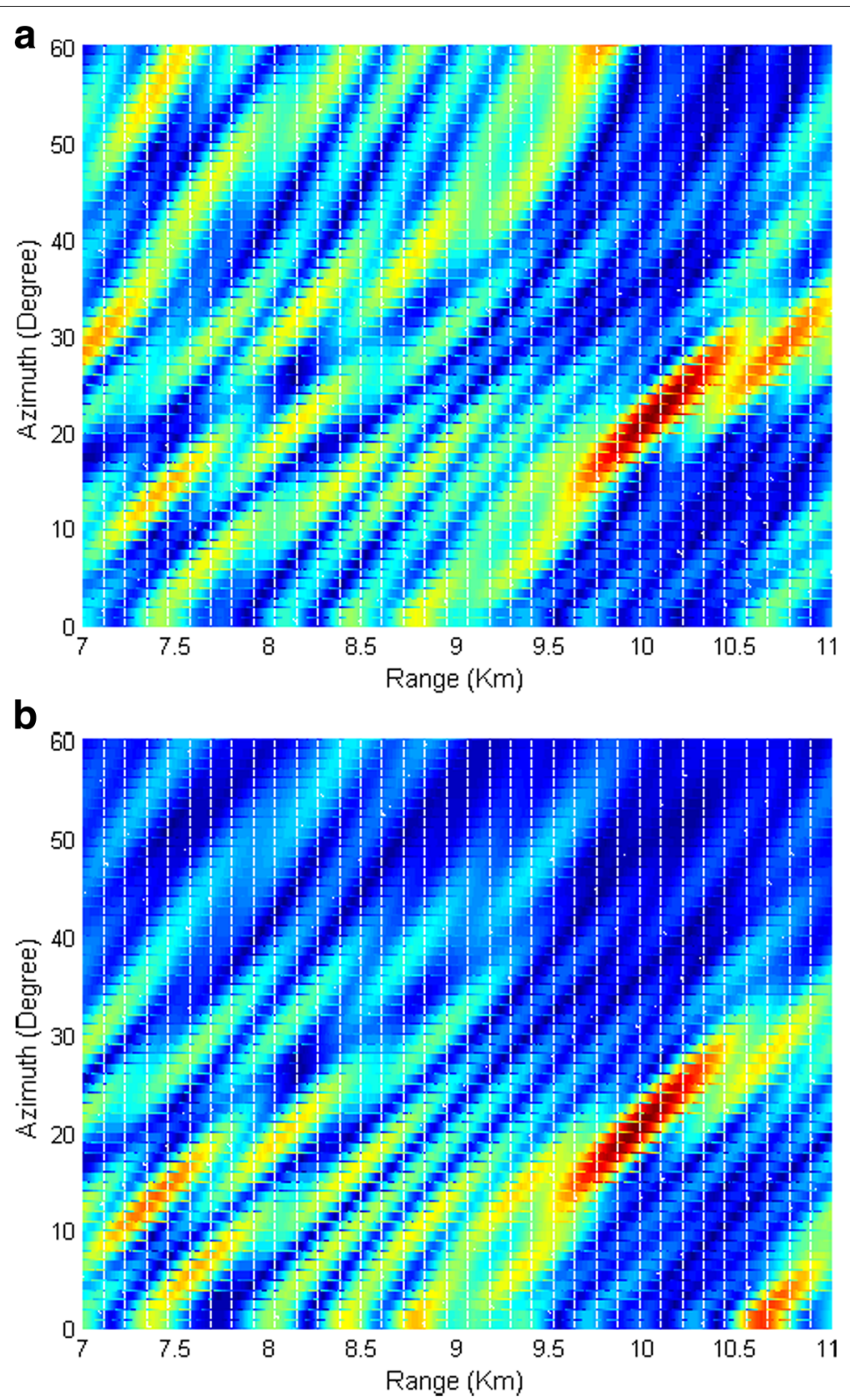

Fig. 6 Angle-range-dependent block-shaped beampattern: a MVAPRS beampattern, b Ours beampattern

tial to focus transmit energy in the desired rangeangle section. More importantly, additional degrees of freedom can be provided if polarized sensors are adopted.

\subsection{Polarization sensitive array}

Considering a right-hand spherical coordinate system with the orthogonal basis defined by orthogonal components $\mathbf{v}_{1}$ and $\mathbf{v}_{2}$ (see Figs. 1 and 2), the measurement model of the vector sensor is given by [27]

$$
\left[\begin{array}{c}
\mathbf{y}_{E}(t) \\
\mathbf{y}_{H}(t)
\end{array}\right]=\left[\begin{array}{c}
\mathbf{I}_{3} \\
(\mathbf{u} \times)
\end{array}\right] \mathbf{V Q h s}(t)+\left[\begin{array}{c}
\mathbf{e}_{E}(t) \\
\mathbf{e}_{H}(t)
\end{array}\right]
$$

where $\mathbf{I}_{3}$ is the third order identity matrix, $(\mathbf{u} \times)=$ $\left[\begin{array}{ccc}0 & -u_{z} & u_{y} \\ u_{z} & 0 & -u_{x} \\ -u_{y} & u_{x} & 0\end{array}\right]$, with $u_{x}, u_{y}, u_{z}$ being the $x, y, z$ components of the unit direction for the propagation vector $\mathbf{u}=[\cos \theta \cos \phi, \sin \theta \cos \phi, \sin \phi]^{T}$. The real-valued matri- 

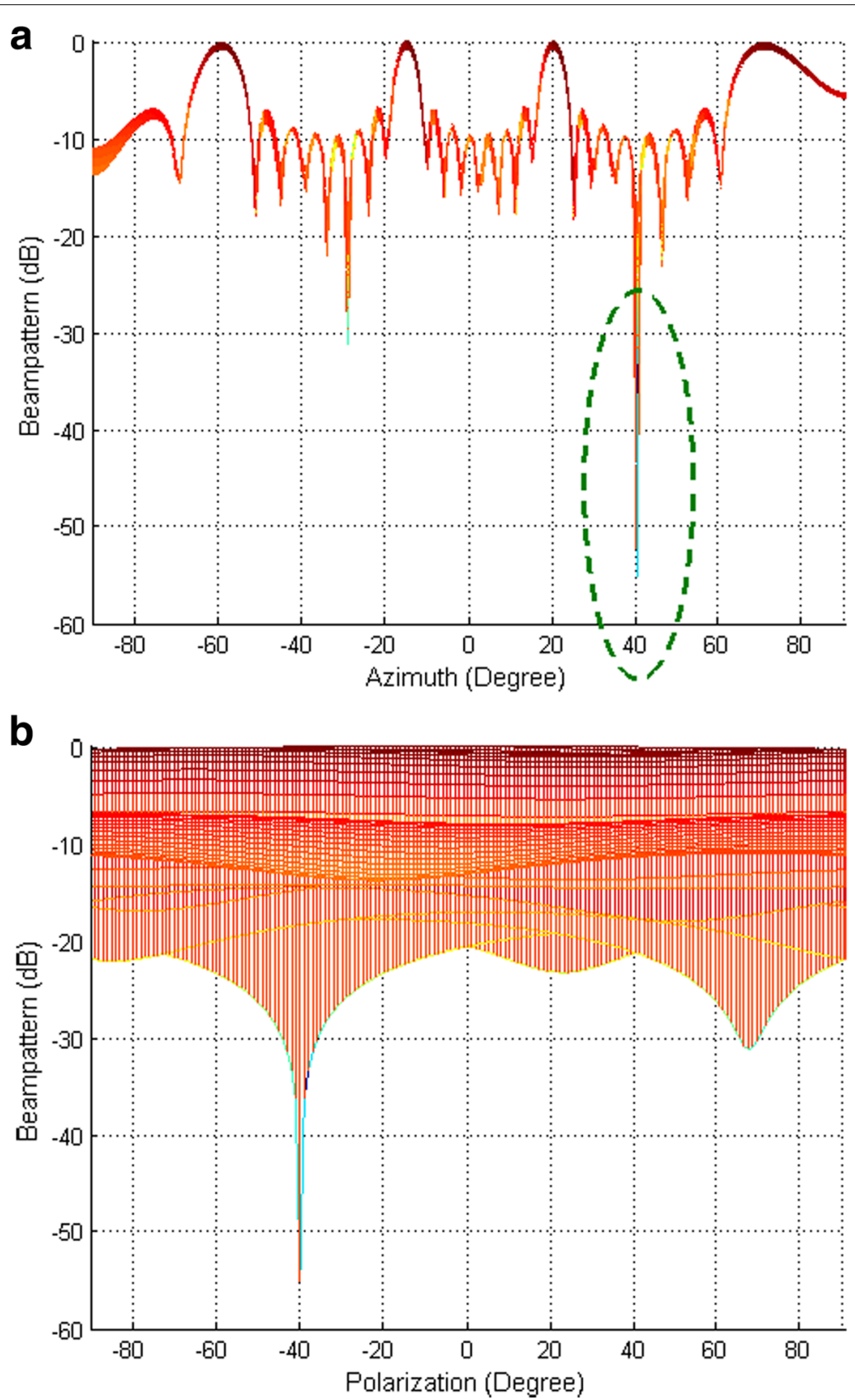

Fig. 7 Angle-polarization-dependent beampattern: a Angle dimension. b Polarization dimension

ces $\mathbf{V} \in \mathcal{R}^{3 \times 2}, \mathbf{Q} \in \mathcal{R}^{2 \times 2}$ and complex vector $\mathbf{h} \in \mathcal{C}^{2 \times 1}$ are given, respectively, by

$$
\begin{aligned}
& \mathbf{V}=\left[\begin{array}{c}
\mathbf{V}_{x}^{(E)} \\
\mathbf{V}_{y}^{(E)} \\
\mathbf{V}_{z}^{(E)}
\end{array}\right]=\left[\begin{array}{cc}
-\sin \theta & -\cos \theta \sin \phi \\
\cos \theta & -\sin \theta \sin \phi \\
0 & \cos \phi
\end{array}\right], \\
& \mathbf{Q}=\left[\begin{array}{cc}
\cos \alpha & \sin \alpha \\
-\sin \alpha & \cos \alpha
\end{array}\right], \mathbf{h}=\left[\begin{array}{c}
\cos \beta \\
j \sin \beta
\end{array}\right],
\end{aligned}
$$

where $\theta \in[0,2 \pi), \phi \in[-\pi / 2, \pi / 2], \alpha \in(-\pi / 2, \pi / 2]$ and $\beta \in[-\pi / 4, \pi / 4]$ are the azimuth, elevation, polarized ellipse's orientation and eccentricity angles, respectively. $\mathbf{s}(t)$ denotes the complex envelope of the transmitted signal. $\mathbf{e}_{E}(t)$ and $\mathbf{e}_{H}(t)$ are the noise components of electric and magnetic fields. $\mathbf{V}_{x}^{(E)}, \mathbf{V}_{y}^{(E)}$, and $\mathbf{V}_{z}^{(E)}$ denote the response of the three electric dipoles along the $x-, y$-, and $z$-axis, respectively. Similarly, the definitions for the three magnetic dipoles along the $x$-, $y$-, and $z$-axis are given by 

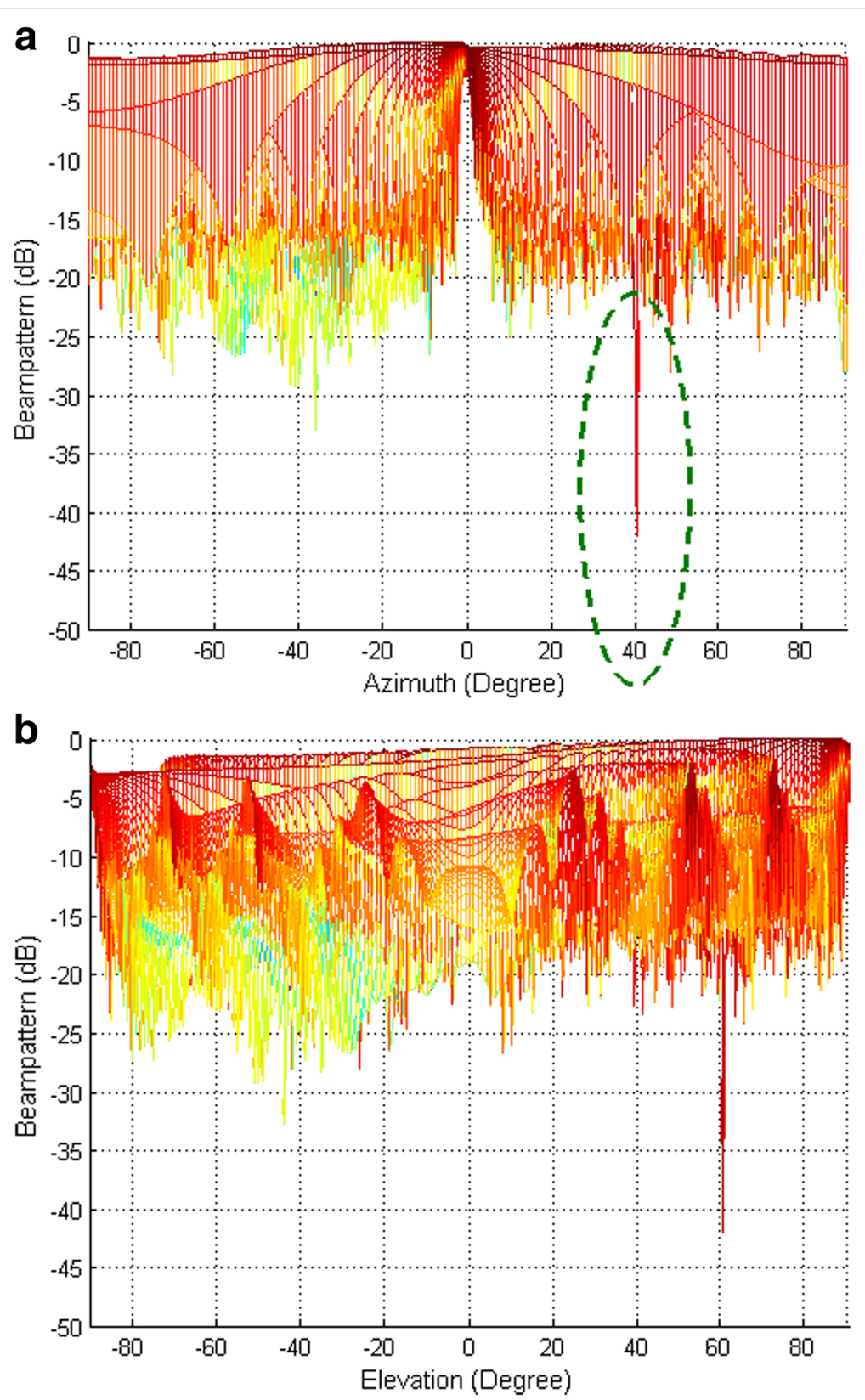

Fig. 8 Azimuth-elevation-dependent beampattern: a Azimuth dimension. b Elevation dimension

$$
\left[\begin{array}{l}
\mathbf{V}_{x}^{(M)} \\
\mathbf{V}_{y}^{(M)} \\
\mathbf{V}_{z}^{(M)}
\end{array}\right]=[\mathbf{u} \times]\left[\begin{array}{l}
\mathbf{V}_{x}^{(E)} \\
\mathbf{V}_{y}^{(E)} \\
\mathbf{V}_{z}^{(E)}
\end{array}\right]=\left[\begin{array}{cc}
-\cos \theta \sin \phi & \sin \theta \\
-\sin \theta \sin \phi & -\cos \theta \\
\cos \phi & 0
\end{array}\right]
$$

Consider a general model of (4), the measurement formulation of a distributed electromagnetic vector sensor (DEMVS), which is also named as distributed electromagnetic component sensor array (see Fig. 3), can be expressed as [28]

$$
\underbrace{\left[\begin{array}{c}
\mathbf{y}_{E}(t) \\
\mathbf{y}_{H}(t)
\end{array}\right]}_{\mathbf{y}(t)}=\sum_{k=1}^{K} \mathbf{a}\left(\Lambda_{k}\right) \mathbf{s}_{k}(t)+\underbrace{\left[\begin{array}{c}
\mathbf{e}_{E}(t) \\
\mathbf{e}_{H}(t)
\end{array}\right]}_{\mathbf{n}(t)}
$$

$$
\mathbf{a}\left(\Lambda_{k}\right)=\Gamma\left(\theta_{k}, \phi_{k}\right) \Omega\left[\begin{array}{c}
\mathbf{I}_{3} \\
\left(\mathbf{u}_{k} \times\right)
\end{array}\right] \mathbf{V}_{k} \mathbf{Q}_{k} \mathbf{h}_{k}
$$

where $\Lambda_{k}=\left[\theta_{k}, \phi_{k}, \alpha_{k}, \beta_{k}\right]$ denotes the direction and polarization parameters of the $k$-th source signal. $\Gamma\left(\theta_{k}, \phi_{k}\right)$ is a diagonal matrix with the $n$-th diagonal entry 


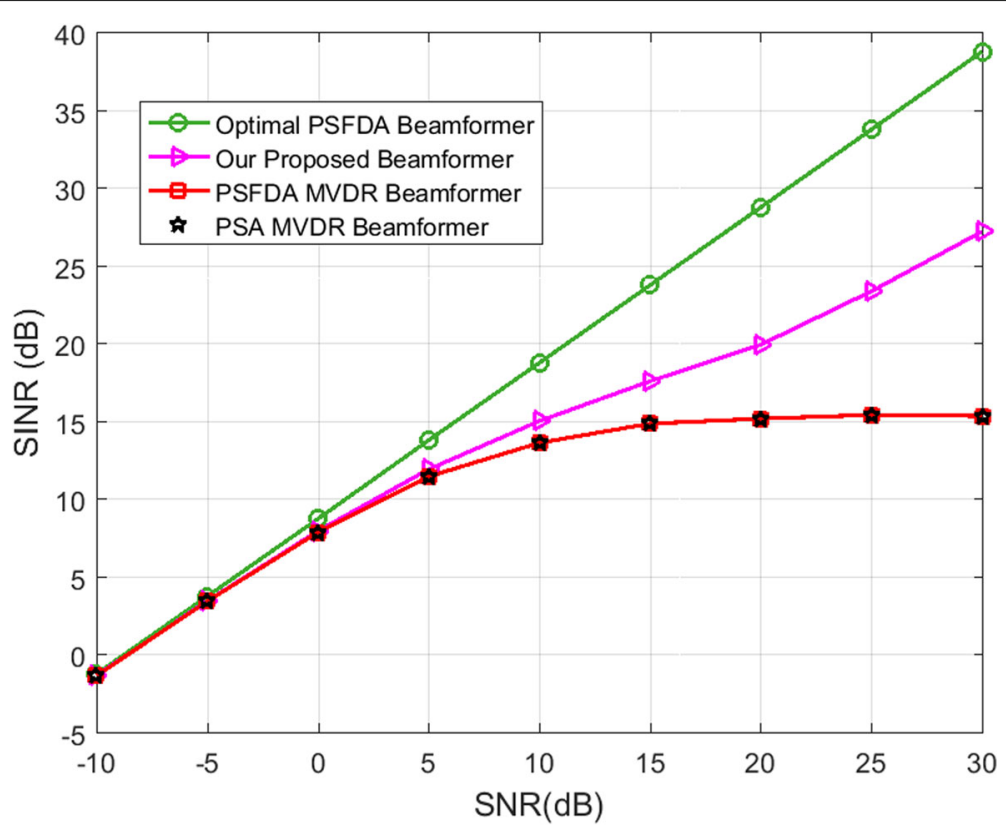

Fig. 9 SINR versus SNR

being $\left[\Gamma\left(\theta_{k}, \phi_{k}\right)\right]_{n}=e^{j 2 \pi \mathbf{q}_{n}^{T} \mathbf{u}_{k} / \lambda} \quad(n=1,2, \ldots, N,(N \leq 6)$ , where $\lambda$ being the wavelength and $N$ is the number of array elements constituting the DEMVS), which provides the phase shift between the DEMVS center and the position $\mathbf{q}_{n}$ of the $n$-th element of the DEMVS, $\Omega$ is an $N \times 6$ selection matrix with elements of " 1 " and " 0 ," indicating the component of the electromagnetic field measured by the $n$-th sensor.
Extending (7) to an array with $M$-DEMVS, we can define the array directional response as $\mathbf{b}\left(\theta_{k}, \phi_{k}\right) \otimes \mathbf{d}\left(\theta_{k}, \phi_{k}\right)$, where $\mathbf{d}\left(\theta_{k}, \phi_{k}\right)=\Gamma\left(\theta_{k}, \phi_{k}\right) \Omega\left[\begin{array}{c}\mathbf{I}_{3} \\ \left(\mathbf{u}_{k} \times\right)\end{array}\right] \mathbf{V}_{k}$ embeds all the directional information of the electromagnetic sources, $\mathbf{b}\left(\theta_{k}, \phi_{k}\right)=\left[e^{j 2 \pi \mathbf{p}_{1}^{T} \mathbf{u}_{k} / \lambda}, e^{j 2 \pi \mathbf{p}_{2}^{T} \mathbf{u}_{k} / \lambda}, \ldots, e^{j 2 \pi \mathbf{p}_{M}^{T} \mathbf{u}_{k} / \lambda}\right]^{T}$ represents the phase of the planewave at the position $\mathbf{p}_{i}$

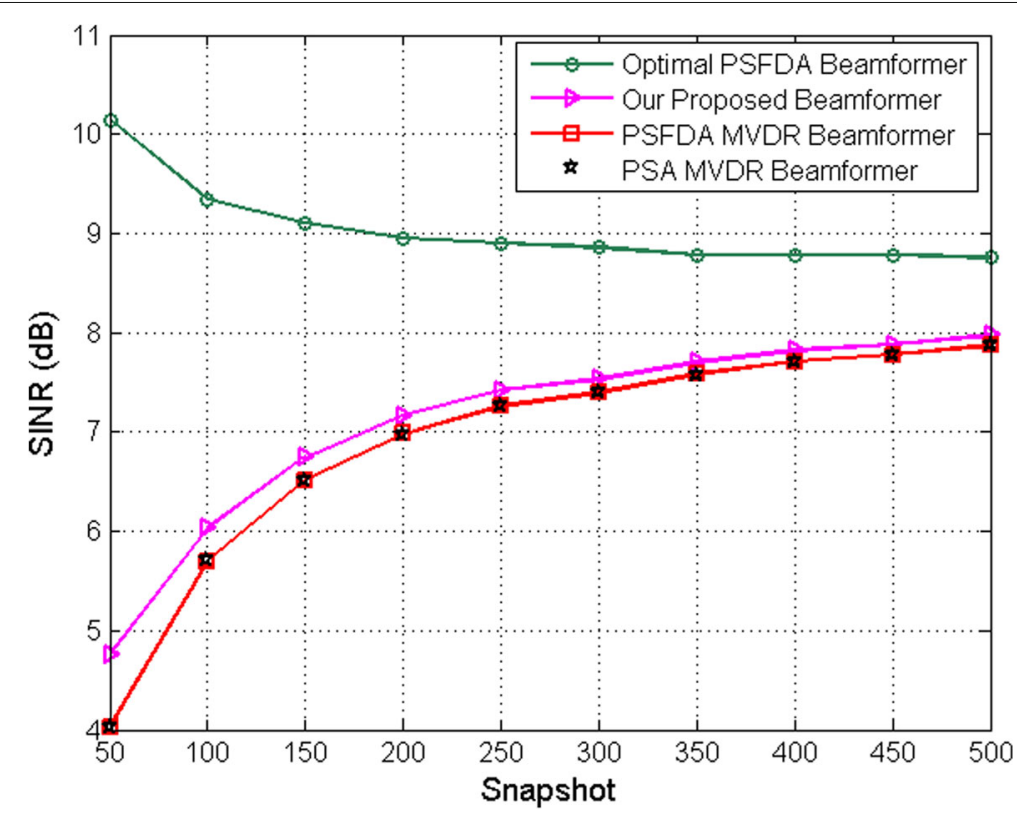

Fig. 10 SINR versus number of snapshots 
Table 1 Vector antenna dipole elements for $2 \leq p \leq 6$ used in the simulations in the Section 4.2

\begin{tabular}{lllllll}
\hline & $\mathbf{V}_{x}^{(E)}$ & $\mathbf{V}_{y}^{(E)}$ & $\mathbf{V}_{z}^{(E)}$ & $\mathbf{V}_{x}^{(M)}$ & $\mathbf{V}_{y}^{(M)}$ & $\mathbf{V}_{z}^{(M)}$ \\
\hline$p=2$ & $\sqrt{ }$ & $\sqrt{ }$ & & & & \\
$p=3$ & $\sqrt{ }$ & $\sqrt{ }$ & $\sqrt{ }$ & & & \\
$p=4$ & $\sqrt{ }$ & $\sqrt{ }$ & $\sqrt{ }$ & $\sqrt{ }$ & $\sqrt{ }$ & $\sqrt{ }$ \\
$p=5$ & $\sqrt{ }$ & $\sqrt{ }$ & $\sqrt{ }$ & $\sqrt{ }$ & $\sqrt{ }$ \\
$p=6$ & $\sqrt{ }$ & $\sqrt{ }$ & $\sqrt{ }$ & $\sqrt{ }$ & $\sqrt{ }$ \\
\hline
\end{tabular}

of the $i$-th DEMVS center $(i=1, \ldots, M)$, and the $\otimes$ is the Kronecker Product. Then, the measurements can be expressed as

$$
\mathbf{y}(t)=\sum_{k=1}^{K}\left[\mathbf{b}\left(\theta_{k}, \phi_{k}\right) \otimes \mathbf{d}\left(\theta_{k}, \phi_{k}\right)\right] \mathbf{Q}_{k} \mathbf{h}_{k} \mathbf{s}_{k}(t)+\mathbf{n}(t) .
$$

According to Kronecker Product relationship $(\mathbf{a} \otimes \mathbf{b}) \mathbf{c}=$ $\mathbf{a} \otimes(\mathbf{b c})$, where $\mathbf{a}$ is a column vector, the number of columns in $\mathbf{b}$ is the same as the number of rows in $\mathbf{c}$. (10) can be rewritten as

$$
\begin{aligned}
\mathbf{y}(t) & =\sum_{k=1}^{K} \mathbf{b}\left(\theta_{k}, \phi_{k}\right) \otimes\left[\mathbf{d}\left(\theta_{k}, \phi_{k}\right) \mathbf{Q}_{k} \mathbf{h}_{k}\right] \mathbf{s}_{k}(t)+\mathbf{n}(t) \\
& =\sum_{k=1}^{K} \mathbf{b}\left(\theta_{k}, \phi_{k}\right) \otimes \mathbf{a}\left(\Lambda_{k}\right) \mathbf{s}_{k}(t)+\mathbf{n}(t)
\end{aligned}
$$

where $\mathbf{a}\left(\Lambda_{k}\right)=\mathbf{d}\left(\theta_{k}, \phi_{k}\right) \mathbf{Q}_{k} \mathbf{h}_{k}$.

Different from the DEMVS, for the EMVS, the dipole elements of the EMVS are collocated, so both $\Gamma$ and $\Omega$ are an $6 \times 6$ identity matrix $\mathbf{I}_{6}$, the $\mathbf{d}\left(\theta_{k}, \phi_{k}\right)$ can be simplified to $\mathbf{d}\left(\theta_{k}, \phi_{k}\right)=\left[\begin{array}{c}\mathbf{I}_{3} \\ \left(\mathbf{u}_{k} \times\right)\end{array}\right] \mathbf{V}_{k}$.

\subsection{Signal model of PSFDA}

For the array constituted by $M$ collocated EMVS, where each vector located at $y$-axis transmits a different frequency. That is, the radiated frequency from the $m$-th EMVS is taken as the same of Eq. 1. Similarly, the phase difference between the m-th EMVS and the first EMVS is

$$
\begin{aligned}
\Delta \psi_{m-1} & =\psi_{m}-\psi_{1} \\
& =-\frac{2 \pi f_{0}(m-1) d \sin \theta \cos \phi}{c}+\frac{2 \pi r(m-1) \Delta f}{c} \\
& -\frac{2 \pi(m-1)^{2} \Delta f d \sin \theta \cos \phi}{c} \\
& \approx-\frac{2 \pi f_{0}(m-1) d \sin \theta \cos \phi}{c}+\frac{2 \pi r(m-1) \Delta f}{c} .
\end{aligned}
$$

Accordingly, the PSFDA signal model can be reformulated as

$$
\begin{aligned}
\mathbf{Z}(t) & =\sum_{k=1}^{K} \mathbf{c}\left(\Lambda_{k}\right) \otimes \mathbf{b}_{a, r}\left(\theta_{k}, \phi_{k}, r_{k}\right) \mathbf{s}_{k}(t)+\mathbf{n}(t) \\
& =\sum_{k=1}^{K} \mathbf{c}\left(\Lambda_{k}\right) \otimes\left[\mathbf{b}_{a, k}\left(\theta_{k}, \phi_{k}\right) \odot \mathbf{b}_{r, k}\left(r_{k}\right)\right] \mathbf{s}_{k}(t)+\mathbf{n}(t) \\
& =\sum_{k=1}^{K} \mathbf{g}\left(\vartheta_{k}\right) \mathbf{s}_{k}(t)+\mathbf{n}(t),
\end{aligned}
$$

where $\mathbf{c}\left(\Lambda_{k}\right)=$

$$
\left[\begin{array}{cc}
-\sin \theta_{k} & -\cos \theta_{k} \sin \phi_{k} \\
\cos \theta_{k} & -\sin \theta_{k} \sin \phi_{k} \\
0 & \cos \phi_{k} \\
-\cos \theta_{k} \sin \phi_{k} & \sin \theta_{k} \\
-\sin \theta_{k} \sin \phi_{k} & -\cos \theta_{k} \\
\cos \phi_{k} & 0
\end{array}\right]\left[\begin{array}{cc}
\cos \alpha_{k} & \sin \alpha_{k} \\
-\sin \alpha_{k} & \cos \alpha_{k}
\end{array}\right]\left[\begin{array}{c}
\cos \beta_{k} \\
j \sin \beta_{k}
\end{array}\right]
$$

$\mathbf{b}_{a, k}\left(\theta_{k}, \phi_{k}\right)=\left[\begin{array}{llll}1 & e^{-j \varphi_{a, k}} & \cdots & e^{-j(M-1) \varphi_{a, k}}\end{array}\right]^{T}$ with $\varphi_{a, k}=$ $2 \pi f_{0} d \sin \theta_{k} \cos \phi_{k} / c, \mathbf{b}_{r, k}\left(r_{k}\right)=\left[\begin{array}{llll}1 & e^{j \psi_{r, k}} & \cdots & e^{j(M-1) \psi_{r, k}}\end{array}\right]^{T}$ with $\psi_{r, k}=2 \pi r_{k} \Delta f / c$, and the $\odot$ is the Khatri-Rao prod$u c t$. In doing so, we get a spatio-polarized-range manifold $\mathbf{g}\left(\vartheta_{k}\right)$, denoting the spatio-polarized-range information $\vartheta_{k}=\left(\theta_{k}, \phi_{k}, \alpha_{k}, \beta_{k}, r_{k}\right)$ of the $k$-th signal. Assume an $x$-electric-component sensor and a $y$-electric-component sensor are used for each vector sensor, namely, the PSFDA array arrangement with uniformly spaced orthogonal dipoles as shown in Fig. 4. In this case, then we have

$$
\begin{aligned}
& \mathbf{c}\left(\Lambda_{k}\right)= \\
& \quad\left[\begin{array}{cc}
-\sin \theta_{k} & -\cos \theta_{k} \sin \phi_{k} \\
\cos \theta_{k} & -\sin \theta_{k} \sin \phi_{k}
\end{array}\right]\left[\begin{array}{cc}
\cos \alpha_{k} & \sin \alpha_{k} \\
-\sin \alpha_{k} & \cos \alpha_{k}
\end{array}\right]\left[\begin{array}{c}
\cos \beta_{k} \\
j \sin \beta_{k}
\end{array}\right] .
\end{aligned}
$$

Similarly, the signal models for the FDA and a conventional uniform linear phased-array (ULA) can be described, respectively, as

$$
\mathbf{z}_{f d a}(t)=\sum_{k=1}^{K} \mathbf{b}_{a, k}\left(\theta_{k}, \phi_{k}\right) \odot \mathbf{b}_{r, k}\left(r_{k}\right) \mathbf{s}_{k}(t)+\mathbf{n}(t),
$$




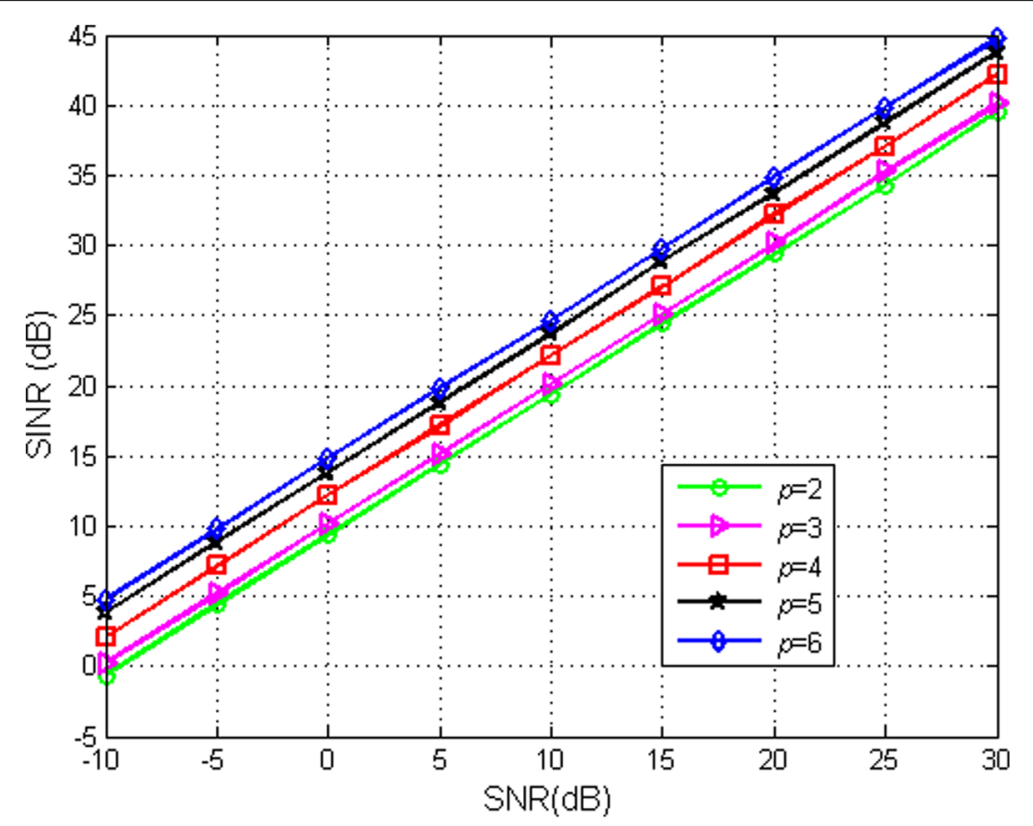

Fig. 11 Optimal SINR versus SNR for antenna dimensionality $p$

$$
\mathbf{Z}_{\text {ula }}(t)=\sum_{k=1}^{K} \mathbf{b}_{a, k}\left(\theta_{k}, \phi_{k}\right) \mathbf{s}_{k}(t)+\mathbf{n}(t) .
$$

The steering vector of PSFDA depends on the angle, range, frequency and polarization, which means the PSFDA has potential advantage in terms of discriminating the targets from interferences.

\section{Proposed adaptive angle, range, and polarization beamformer}

The PSFDA measurement model for the $M$ collocated EMVS can be expressed as

$$
\mathbf{Z}(t)=\mathbf{g}\left(\vartheta_{s}\right) \mathbf{s}(t)+\sum_{j=1}^{J} \mathbf{g}\left(\vartheta_{j}\right) \mathbf{i}_{j}(t)+\mathbf{n}(t), t=1, \ldots, T
$$

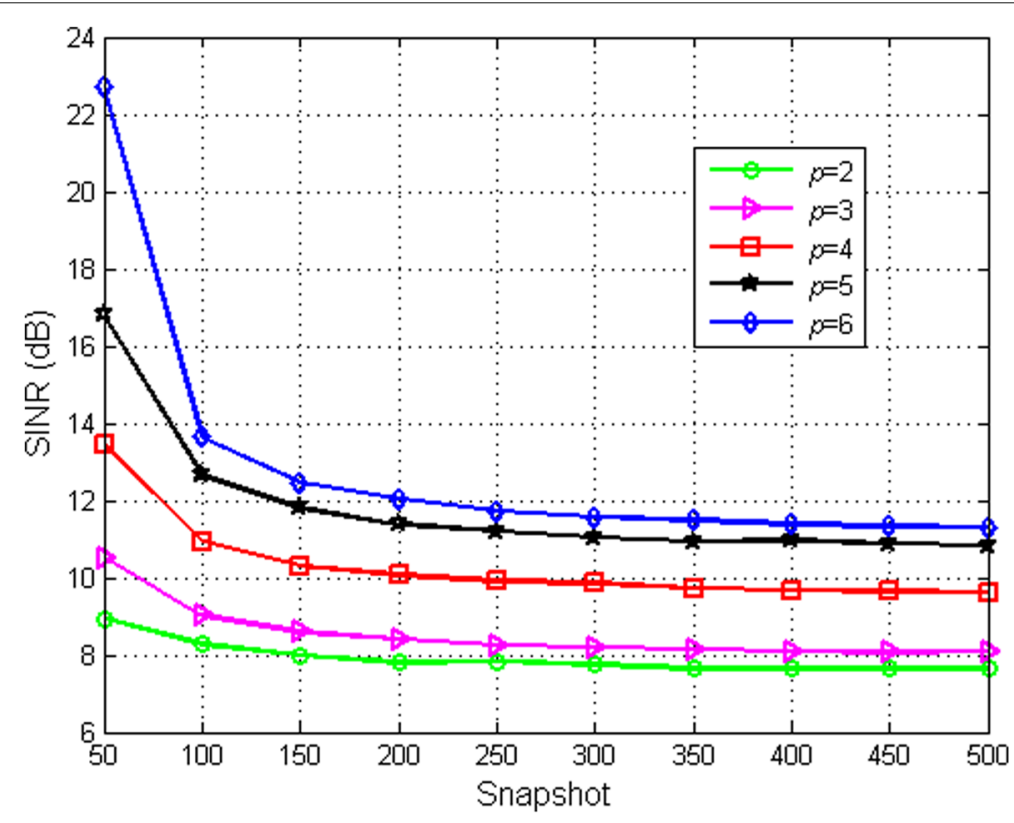

Fig. 12 Optimal SINR versus number of snapshots for antenna dimensionality $p$ 
where $\mathbf{g}\left(\vartheta_{s}\right)$ and $\mathbf{g}\left(\vartheta_{j}\right)(j=1, \ldots, J=K-1)$ are the steering vectors for signal and interference, respectively. In the following, we assume that the signal of interest is $\mathbf{s}(t)$, interferences is $\mathbf{i}_{j}(t)(j=1, \ldots, J)$ and $\mathbf{n}(t)$ is a zero mean white Gaussian process.

According to the minimum-noise-variance beamformer for PSA [29], we can get the classical minimum variance for the angle-polarization-range sensitive (MVAPRS) beamformer as

$$
\mathbf{w}_{d}=\frac{\mathbf{R}_{z}^{-1} \mathbf{g}\left(\vartheta_{s}\right)}{\mathbf{g}^{H}\left(\vartheta_{s}\right) \mathbf{R}_{z}^{-1} \mathbf{g}\left(\vartheta_{s}\right)},
$$

where $\mathbf{R}_{z}=E\left\{\mathbf{Z}(t) \mathbf{Z}^{H}(t)\right\}$ denotes the $2 M \times 2 M$ covariance matrix of the array snapshot vectors, and $(\cdot)^{-1}$ indicates the matrix inversion operator. To further enhance the interference mitigation capability, we propose an adaptive angle-range-polarization-dependent beamforming method. For a small number of interferences in the sidelobe domain, and non-sparse array gains in the main-lobe, to make the sidelobe of beampattern sparse, we sample the whole observation domain and form a overcomplete basis $\mathbf{G}_{s}=$ $\left[\mathbf{g}\left(\bar{\vartheta}_{1}\right), \mathbf{g}\left(\bar{\vartheta}_{2}\right), \ldots, \mathbf{g}\left(\bar{\vartheta}_{s-q}\right), \mathbf{g}\left(\bar{\vartheta}_{s+q}\right), \ldots \mathbf{g}\left(\bar{\vartheta}_{L_{0}}\right)\right]\left(L_{0} \gg\right.$ $K)$ corresponding to the sidelobe region of $\vartheta_{s}$, where q is an integer associate with the bounds between the mainlobe and sidelobes of the beampattern, and we add the $\ell_{1}$-norm penalization on the array gains $\mathbf{G}_{s}$. To further suppress the sidelobe level and interference, the $\ell_{\infty}$-norm penalization is further imposed on the $\mathbf{G}_{s}$. In doing so, the proposed beamformer $\mathbf{w}$ can be recast as,

$$
\begin{aligned}
& \underset{\mathbf{w}}{\operatorname{minimize}} \mathbf{w}^{H} \mathbf{R}_{z} \mathbf{w}+\xi\left\|\mathbf{w}^{H} \mathbf{G}_{s}\right\|_{1} \\
& \text { subject to }\left\|\mathbf{w}^{H} \mathbf{g}\left(\varphi_{s}\right)-1\right\|_{\infty} \leq \varepsilon \\
& \|\| \mathbf{w}^{H} \mathbf{G}_{s} \|_{\infty} \leq \eta
\end{aligned}
$$

where the weighting factor $\xi$ makes a tradeoff between the minimum variance constraint on total output energy and the sparse constraint on the sidelobe of beampattern, the parameters $\varepsilon$ and $\eta$ are designed according to the desired array performance. So the array gain including both sidelobes and interferences can be suppressed by $\left\|\mathbf{w}^{H} \mathbf{G}_{s}\right\|_{\infty} \leq$ $\eta$. The $\left\|\mathbf{w}^{H} \mathbf{g}\left(\varphi_{s}\right)-1\right\|_{\infty} \leq \varepsilon$ in (Eq. 18) aims to maintain the signal of interest. $\|\mathbf{c}\|_{\hbar}^{\hbar}=\sum_{i}\left|c_{i}\right|^{\hbar}$ is $\ell_{\hbar}$-norm of the vector $\mathbf{c}, \hbar \leq 1$ leads to sparse solutions, while $\hbar=2$ is the $\ell_{2}$-norm criterion. Due to the sparsity of the beam pattern,and to make the optimization problem (Eq. 18) be an easy-to-handle convex problem, we choose $\hbar=1$, that is $\left\|\mathbf{w}^{H} \mathbf{G}_{s}\right\|_{1}$. Compared with the $\ell_{2}$-norm criterion that favors solutions with many nonzero entries, this sparse constraint has advantages for the cases with few nonzero elements for interference suppression and sidelobe reduction. The validity of our proposed method will be further demonstrated via numerical analysis in Section 4.

\section{Simulation Results and Discussions}

We first analyze the PSFDA performance by examing its spatial domain beampattern, spatial-polarization beampattern and spatial-range beampattern. Statistical simulations are carried out in an ideal scenario without steering vector mismatches.

In the following simulations, we consider a PSFDA with $M=8$ orthogonal dipole pairs and $d=0.5 \mathrm{~m}$ as shown in the Fig. 4. Suppose there are one signal of interest and one interference with fixed signal-to-interference ratio (SIR) equal to $-20 \mathrm{~dB}, 100$ independent runs in spatially white gaussian noise. We assume that the powers of the polarized signal, interference and noise are $\sigma_{s}^{2}, \sigma_{j}^{2}$ and $\sigma_{n}^{2}$, respectively. The average SINR is determined by:

$$
\mathbf{S I N R}=\frac{\sigma_{s}^{2} \mathbf{w}^{\mathbf{H}} \mathbf{g}\left(\vartheta_{\mathbf{s}}\right) \mathbf{g}^{\mathbf{H}}\left(\vartheta_{\mathbf{s}}\right) \mathbf{w}}{\sum_{j=1}^{J} \sigma_{j}^{2} \mathbf{g}\left(\vartheta_{j}\right) \mathbf{g}^{H}\left(\vartheta_{j}\right)+\sigma_{n}^{2} \mathbf{I}}
$$

where $\mathbf{I} \in \mathcal{C}^{2 M \times 2 M}$.

\subsection{Beampattern}

\subsubsection{Angle-range-dependent beampattern}

Suppose the signal and interference have the anglerange-polarization characteristics $\left(\theta_{s}, \phi_{s}, \alpha_{s}, \beta_{s}, r_{s}\right)=$ $\left(20^{\circ}, 60^{\circ},-20^{\circ}, 30^{\circ}, 10 \mathrm{~km}\right)$ and $\left(\theta_{j}, \phi_{j}, \alpha_{j}, \beta_{j}, r_{j}\right)=$ $\left(20^{\circ}, 60^{\circ},-20^{\circ}, 30^{\circ}, 8 \mathrm{~km}\right)$, respectively. For an ideal scenario with the exact knowledge of the steering vector, Fig. 5 compares the PSA and PSFDA beampatterns. The angle-range-dependent $S$-shaped PSFDA beampattern has additional degrees of freedom and potential applications in suppressing the range interference and ambiguous clutter with same angle but different range from the target, while the range-independent PSA beampattern has no such advantages. Besides, compared with PSFDA, the FDA does not provide the polarization information.

We also compare the block-shaped anglerange-dependent beampattern obtained by the MVAPRS beamformer and our proposed beamformer for the PSFDA with the frequency offsets as $\Delta f_{m}=(m-1)^{2} \cdot \Delta f, m=1,2, \ldots, M$. The results given in Fig. 6 demonstrate that our proposed algorithm can successfully suppress the interference as well as the MVAPRS.

\subsubsection{Spatio-polarized beampattern}

We compare the PSFDA angle-polarization-dependent beampattern and the angle-elevation-dependent beampattern like that of a PSA beampattern, as shown in the Figs. 7 and 8, respectively. From Fig. 7, It is seen that the angle-polarization-dependent beampattern can keep the maximum energy at the target positions $\left(20^{\circ}, 60^{\circ}, 20^{\circ}, 30^{\circ}, 10 \mathrm{~km}\right)$ and suppress simultaneously the interference from $\left(40^{\circ}, 60^{\circ},-40^{\circ}, 30^{\circ}, 10 \mathrm{~km}\right)$. 


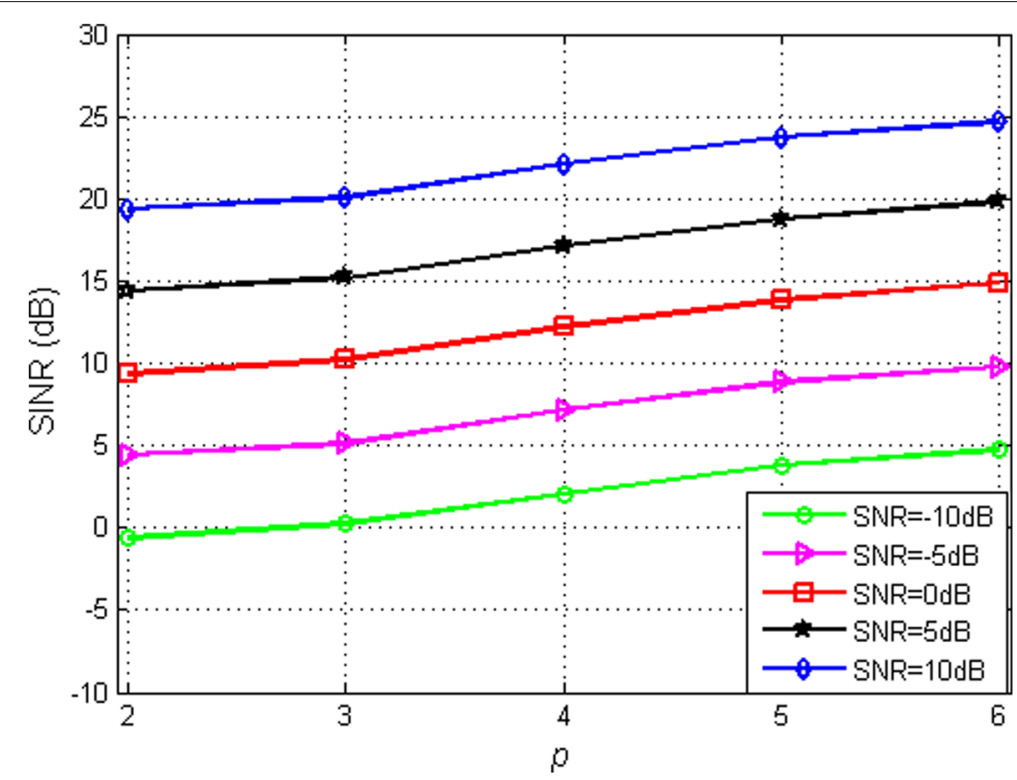

Fig. 13 Optimal SINR versus antenna dimensionality $p$ for SNR

Similarly, the angle-elevation-dependent beampattern effectively suppresses the interference from $\left(40^{\circ}, 60^{\circ},-20^{\circ}, 30^{\circ}, 10 \mathrm{~km}\right)$, without reducing energy at the target $\left(20^{\circ}, 30^{\circ},-20^{\circ}, 30^{\circ}, 10 \mathrm{~km}\right)$. Moreover, the PSFDA not only has the same performance as that of PSA, but the former also outperforms the latter in range-dimension, as shown in Fig. 5.
In addition, we consider the statistical results for the angle-range-dependent block-shaped beampattern. Fig. 9 displays the output SINRs versus signal-to-interference ratio (SNR) for $T=500$. For a fixed SNR $=0 \mathrm{~dB}$, the SINRs versus the number of snapshots $T$ are shown in Fig. 10. The results clearly demonstrate that our proposed beamformer substantially outperforms the MVAPRS for SNR >

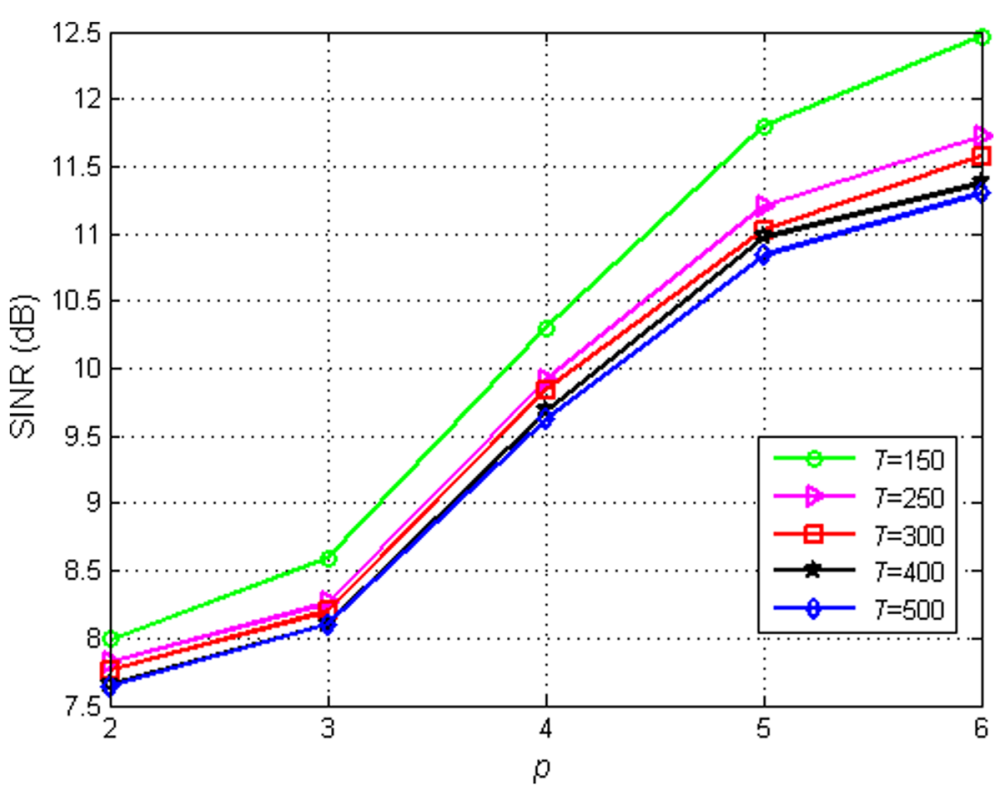

Fig. 14 Optimal SINR versus antenna dimensionality $p$ for number of snapshots 
OdB. Since the proposed algorithm is a convex optimization problem, it can be efficiently resolved by the openly CVX software [30].

\subsection{High-dimensional arrays}

Finally, we analyze the performance of the optimal output SINR for high-dimensional arrays. The angle-rangepolarization characteristics are same as that of Fig. 5 . In each antenna, we consider the antenna elements with $2 \leq p \leq 6$. For each $p$, the dipole elements are chosen according to Table 1 . Note that at each row, the symbol " $\sqrt{ }$ " denotes that a certain dipole element is selected in the antenna. For example, when $p=2$, two electric dipoles at the $x$-axis and $y$-axis are selected, respectively. Accordingly, the antenna response is $\left[\begin{array}{c}\mathbf{V}_{x}^{(E)} \\ \mathbf{V}_{y}^{(E)}\end{array}\right]$.

The simulated optimal output SINRs versus SNR for $p=2,3,4,5,6$, under the angle-range-dependent blockshaped beampattern, are given in Fig. 11. For fixed $\mathrm{SNR}=0 \mathrm{~dB}$ and $\mathrm{SIR}=-20 \mathrm{~dB}$, the optimal SINRs versus $T$ for $p=2,3,4,5,6$ are shown in Fig. 12. It is noticed along with the increase of the sensor dimensionality $p$, the output SINRs will increase even under the same SNR (or number of snapshots). Figures 13 and 14 show that the optimal output SINR is approximately linearly proportional to $p$. This verify that the electromagnetic vector array has the advantage of enabling the control of beampattern polarization, which achieves more degrees of freedom.

\section{Conclusions}

In this paper, we formulated the PSFDA signal model and proposed a corresponding beamformer based on the MVAPRS, $\ell_{1}$-norm minimization and $\ell_{\infty}$-norm constraint. The proposed adaptive beamformer is superior to the conventional MVAPRS on maintaining array gain for the signal of interest, and PSA beamformers in terms of both nulling interferences and maintaining signal of interest. In addition, the performance of the optimal output SINR is also analyzed for the high-dimensional arrays. Simulation results demonstrate that the electromagnetic vector array has the advantage of enabling the control of beampattern polarization and virtually increasing the array size.

\section{Abbreviations \\ DEMVS: Distributed electromagnetic vector sensor; DOA: Direction-of-arrival; EMVS: Electromagnetic vector sensor; FDA: Frequency diverse array; MIMO: Multi-input and multi-output; MVAPRS: Minimum variance beamformer for the angle-polarization-range sensitive; PSA: Polarization sensitive array; PSFDA: Polarization sensitive frequency diverse array; SINR: signal-to-interference-plus-noise ratio; SIR: signal-to-interference ratio; SNR: signal-to-noise ratio}

\section{Acknowledgements}

This work was supported in part by the National Natural Science Foundation of China (61501089 \& 61471103), Sichuan Science and Technology Program (18ZDYF2551), and Fundamental Research Funds for the Central Universities (ZYGX2018J005).

\section{Funding}

No sources of funding for the research reported.

\section{Availability of data and materials}

Data sharing not applicable to this article as no datasets were generated or analysed during the current study.

\section{Authors' contributions}

$\mathrm{HC}$ formulated the idea and wrote the paper, all authors discussed the idea together. Additionally, all authors read and approved the final manuscript.

\section{Ethics approval and consent to participate}

Manuscripts reporting studies do not involve human participants, human data or human tissue.

\section{Consent for publication}

This manuscript does not contain any individual person's data in any form.

\section{Competing interests}

The authors declare that they have no competing interests.

\section{Publisher's Note}

Springer Nature remains neutral with regard to jurisdictional claims in published maps and institutional affiliations.

\section{Author details}

${ }^{1}$ School of Information and Communication Engineering, University of Electronic Science and Technology of China, Xiyuan Road, Chengdu, 611731, People's Republic of China. ${ }^{2}$ The University of Tokyo, Tokyo, Japan.

Received: 22 August 2018 Accepted: 27 March 2019

Published online: 11 April 2019

\section{References}

1. H. Hong, X. P. Mao, C. Hu, in Proceedings of the IEEE Radar Conference, Atlanta, GA, USA. A multi-domain collaborative filter for HFSWR based on oblique projection (IEEE, 2012), pp. 907-912

2. X. P. Mao, Y. T. Liu, Null phase-shift polarization filtering for high-frequency radar. IEEE Trans. Aerosp. Electron. Syst. 43(4), 1397-1408 (2007)

3. X. P. Mao, A. J. Liu, H. J. Hou, H. Hong, R. Guo, W. B. Deng, Oblique projection polarisation filtering for interference suppression in high-frequency surface wave radar. IET Radar Sonar Navig. 6(2), 71-80 (2012)

4. J. Ward, in Proceedings of the IEEE International Conference on Acoustics, Speech and Signal Processing, Detroit, MI, USA. Space-time adaptive processing for airborne radar (IEEE, 1995), pp. 2809-2812

5. P. Antonik, M. C. Wicks, H. D. Griffiths, C. J. Baker, in Proceedings of the IEEE Conference on Radar, Syracuse, NY, USA. Frequency diverse array radars (IEEE, 2006), pp. 215-217

6. M. C. Wicks, P. Antonik, Frequency diverse array with independent modulation of frequency, amplitude, and phase., U.S. Patent 7,319,427B2 (2008)

7. M. C. Wicks, P. Antonik, Method and Apparatus for a Frequency Diverse Array., U.S. Patent 7,511,665B2 (2009)

8. S. Mustafa, D. Simsek, H. Altunkan, E. Taylan, in Proceedings of the IEEE Conference on Radar, Waltham, MA, USA. Frequency diverse array antenna with periodic time modulated pattern in range and angle (IEEE, 2007), pp. 427-430

9. P. Antonik, M. C. Wicks, H. D. Griffiths, C. J. Baker, in Proceedings of the International Waveform Diversity and Design Conference, Kauai, Hawaii, USA. Range dependent beamforming using element level waveform diversity (IEEE, 2006), pp. 71-76

10. W.-Q. Wang, Range-angle dependent transmit beampattern synthesis for linear frequency diverse arrays. IEEE Trans. Antennas Propag. 61(8), 4073-4081 (2013)

11. Y. Xu, X. Shi, J. Xu, P. Li, Range-angle-dependent beamforming of pulsed frequency diverse array. IEEE Trans. Antennas and Propag. 63(7), 3262-3267 (2015)

12. A. Basit, L. M. Qureshi, W. Khan, S. U. khan, in Proceedings of the 12th International Bhurban Conference on Applied Sciences and Technology 
(IBCAST), Islamabad, Pakistan. Cognitive frequency offset calculation for frequency diverse array radar (IEEE, 2015), pp. 641-645

13. W.-Q. Wang, H. C. So, H. Shao, Nonuniform frequency diverse array for range-angle imaging of targets. IEEE Sensors J. 14(8), 2469-2476 (2014)

14. W. Q. Wang, H. Shao, Range-angle localization of targets by a double-pulse frequency diverse array radar. IEEE J. Sel. Top. Sig. Process. 8(1), 106-114 (2014)

15. P. F. Sammartino, C. J. Baker, H. D. Griffiths, Frequency diverse MIMO techniques for radar. IEEE Trans. Aerosp. Electron. Syst. 49(1), 201-222 (2013)

16. W. Q. Wang, Phased-MIMO radar with frequency diversity for range-dependent beamforming. IEEE Sensors J. 13(4), 1320-1328 (2013)

17. W. Khan, I. M. Qureshi, A. Basit, M. Zubair, A double pulse MIMO frequency diverse array radar for improved range-angle localization of target. Wirel. Pers. Commun. 82(4), 1-15 (2015)

18. J. Xu, S. Zhu, G. Liao, Space-time-range adaptive processing for airborne radar systems. IEEE Sensors J. 15(3), 1602-1610 (2015)

19. W. Khan, I. Qureshi, Frequency diverse array radar with time-dependent frequency offset. IEEE Antennas Wirel. Propag. Lett. 13, 758-761 (2014)

20. W. Khan, I. M. Qureshi, S. Saeed, Frequency diverse array radar with logarithmically increasing frequency offset. IEEE Antennas Wirel. Propag. Lett. 14, 499-502 (2014)

21. Y. B. Wang, W. Q. Wang, H. Z. Shao, Frequency diverse array Cramer-Rao lower bounds for estimating direction, range and velocity. Int. J. Antennas Propag. 2014, 106-114 (2014)

22. C. Cetintepe, S. Demir, Multipath characteristics of frequency diverse arrays over a ground plane. IEEE Trans. Antennas and Propag. 62(7), 3567-3574 (2014)

23. A. M. Jones, B. D. Rigling, in Proceedings of the IEEE Radar Conference, Atlanta, GA, USA. Planar frequency diverse array receiver architecture, (2012), pp. 145-150

24. D. L. Donoho, Compressed sensing. IEEE Trans. Inf. Theory. 52(4), 1289-1306 (2006)

25. S. Boyd, L. Vandenberghe, Convex Optimization. (Cambridge university press, Cambridge, 2004)

26. H. Chen, H. Shao, W. Wang, in Proceedings of the IEEE International Conference on Acoustics, Speech and Signal Processing, Shanghai, China. Sparse reconstruction-based angle-range-polarization-dependent beamforming with polarization sensitive frequency diverse array (IEEE, 2016), pp. 2891-2895

27. A. Nehorai, E. Paldi, Vector-sensor array processing for electromagnetic source localization. IEEE Trans. Sig. Process. 42(2), 376-398 (1994)

28. C. See, A. Nehorai, in Proceedings of the 7th Workshop on Adaptive Sensor Array Processing, Boston, Massachusetts, USA. Distributed electromagnetic component sensor array processing, (1999), pp. 75-79

29. A. Nehorai, K. C. Ho, B. T. G. Tan, Minimum-noise-variance beamformer with an electromagnetic vector sensor. IEEE Trans. Sig. Process. 47(3), 601-618 (1999)

30. M. Grant, S. Boyd, Y. Ye, cvx users' guide for cvx version 1.1. accessiable at: (2014). http://www.stanford.edu/ boyd/index.html. Accessed 21 Jan 2014

\section{Submit your manuscript to a SpringerOpen ${ }^{\circ}$ journal and benefit from:}

- Convenient online submission

- Rigorous peer review

- Open access: articles freely available online

- High visibility within the field

- Retaining the copyright to your article 